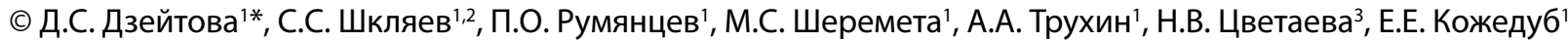

\author{
${ }^{1}$ Национальный медицинский исследовательский центр эндокринологии, Москва, Россия \\ ${ }^{2}$ Национальный медицинский исследовательский центр радиологии МРНЦ им. А.Ф. Цыба, Обнинск, Россия \\ ${ }^{3}$ Национальный медицинский исследовательский центр гематологии, Москва, Россия
}

В данной работе описан клинический случай лечения пациентки с тиреотоксикозом при сопутствующей гематологической патологии в виде носительства нестабильного гемоглобина Hasharon. Пациентка с диагнозом «Диффузноузловой токсический зоб. Тиреотоксикоз средней степени тяжести. Медикаментозный гипотиреоз» поступила в отделение радионуклидной терапии с целью проведения лечения радиоактивным йодом. Дебют заболевания - лето 2018 г. (тиреотропный гормон (ТТГ) - 0 мМЕ/мл). На диагностическом этапе перед проведением радиойодтерапии (РЙТ) выполнены УзИ и сцинтиграфия щитовидной железы. Из анамнеза известно, что в 2000 г. у пациентки было подозрение на наличие аномального гемоглобина, с тех пор обследований не проводилось и анемии никогда не выявлялось. При подготовке к РЙТ был верифицирован диагноз наследственной гемоглобинопатии с наличием (17\%) нестабильного гемоглобина Hasharon-Sinai-Sealy в гетерозиготной форме. 02.07.2019 проведена радионуклидная терапия I131 активностью 400 МБк. В посттерапевтическом периоде проводили ежемесячный контроль лабораторных и инструментальных показателей: состояние гипотиреоза достигнуто к концу 2 месяца после РЙТ, за период наблюдения не было отмечено эпизодов значительного повышения уровня билирубина; побочных эффектов от проведения РЙТ не зафиксировано. На примере вышеописанного наблюдения можно судить о безопасности проведения РЙТ тиреотоксикоза у пациентов со схожими гемоглобинопатиями, не исключая, однако, необходимости индивидуального подхода в каждом конкретном случае.

КЛЮЧЕВЫЕ СЛОВА: тиреотоксикоз, радиойодтерапия, гемоглобинопатия Hasharon, клинический случай, сцинтиграфия.

\section{RADIOIODINE THERAPY OUTCOME IN TOXIC MULTINODULAR GOITER PATIENT WITH CONCOMITANT HEREDITARY HASHARON HEMOGLOBINOPATHY}

(c) Dali S. Dzeytova ${ }^{1 *}$, Stanislav S. Shklyaev ${ }^{1,2}$, Pavel O. Rumyantsev¹, Marina S. Sheremeta', Alexey A.Trukhin', Nina V.Tsvetaeva ${ }^{3}$, Evgenii E. Kozhedub

\footnotetext{
${ }^{1}$ Endocrinology Research Centre, Moscow, Russia

${ }^{2}$ National Medical Research Center for Radiology, MRRC n.a. A.F. Tsyb, Obninsk, Russia

${ }^{3}$ National Research Center for hematology, Moscow, Russia
}

This research describes a clinical case of treatment of a patient with thyrotoxicosis with concomitant hematological pathology - carriage of unstable hemoglobin Hasharon. A patient diagnosed with «Diffuse toxic nodular goiter. Thyrotoxicosis of medium severity. Drug-induced hypothyroidism» was admitted to the Department of radionuclide therapy for the purpose of treatment with radioactive iodine. Onset of disease - summer 2018 (thyroid-stimulating hormone (TSH) $0 \mathrm{mlU} / \mathrm{ml}$ ). The instrumental studies (ultrasound, scintillation scanning of the thyroid gland) were performed at the preradioiodine therapy (RIT) diagnostic stage. The history of the disease indicates, that in 2000 the patient was suspected of having abnormal hemoglobin, since then no examinations have been conducted and anemia has never been detected. The diagnosis of ancestral hemoglobinopathy with the presence (17\%) of unstable Hasharon-Sinai-Sealy hemoglobin in a heterozygous form was verified during the preparation to RIT. The radionuclide therapy I $^{131}$ with activity of $400 \mathrm{MBq}$ was performed on 02.07.2019. The monthly monitoring of laboratory and instrumental indicants was carried out during the post-therapeutic period: the state of hypothyroidism was reached by the end of 2 months after RT, no episodes of significant increase in bilirubin levels were observed during the observation period; no side effects from RT were stated. It becomes possible based on the example of the above observation, to judge the safety of conducting RT for treatment of thyrotoxicosis in patients with similar hemoglobinopathy, without excluding, however, the need for an individual approach in each case.

KEYWORDS: thyrotoxicosis, radioiodine therapy, hemoglobinopathy Hasharon, unstable hemoglobin, scintigraphy. 


\section{АКТУАЛЬНОСТЬ}

Гипертиреоз - это патологическое состояние, при котором щитовидной железой (ЩЖ) синтезируется и секретируется избыточное количество гормонов. Стойкое повышенное содержание свободного тироксина (свТ4) и/или свободного трийодтиронина (свТ3) и их токсическое воздействие на различные органы и ткани приводят к развитию синдрома тиреотоксикоза. При лабораторном исследовании данного состояния наблюдают низкую концентрацию ТТГ (<0,1 мЕ/л) и повышенное содержание в сыворотке свТ4 и свТ3, что расценивают как «манифестный» тиреотоксикоз; также стоит отметить возможность «субклинического» тиреотоксикоза, при котором снижение уровня ТТГ не сопровождается одновременным повышением концентрации тиреоидных гормонов в крови $[1,2]$.

Распространенность гипертиреоза у женщин составляет от 0,5 до 2\%, что примерно в 10 раз чаще, чем у мужчин [3]. В регионах с нормальным йодным обеспечением наиболее частой причиной тиреотоксикоза является диффузный токсический зоб, тогда как в йододефицитных регионах в этиологической структуре токсического зоба болезнь Грейвса конкурирует с функциональной автономией ЩЖ [4].

Тяжесть заболевания определяется его обширной клинической картиной: повышенная возбудимость, эмоциональная лабильность, беспокойство, нарушение сна, нарушение концентрации внимания, слабость, потливость, сердцебиение, уменьшение массы тела; наиболее грозными являются сердечно-сосудистые осложнения, в особенности у лиц пожилого возраста $[5,6]$.

Консервативное лечение принято считать первой линией терапии тиреотоксикоза, препараты выбора относятся к группе тиреостатиков. Однако, учитывая возможные побочные эффекты длительной тиреостатической терапии, сложность контроля и риск рецидива заболевания после отмены тиреостатика, достигающий 70\% и более, все большее количество специалистов отдают предпочтение радикальным методам лечения заболевания - хирургии и терапии радиоактивным йодом $[2,7]$.

Эффективность хирургического лечения не вызывает сомнений, но оперативное вмешательство сопряжено с риском повреждения возвратного гортанного нерва во время операции, развитием послеоперационного гипопаратиреоза. При этом исход операции во многом зависит от квалификации хирурга.

Другим радикальным методом лечения является РЙТ. Механизм РЙТ основан на избирательном накоплении до 65\% всего введенного радиофармпрепарата (РФП), меченного радиоактивным 131-йодом, обладающего оптимальными характеристиками: период полураспада 8 дней, гамма-спектр подходит для регистрации системами однофотонная эмиссионная компьютерная томография, дешевизна производства. Порядка 92-98\% энергии бета-частиц поглощается в тиреоцитах щЖ, 2-8\% распределяются в пределах 1-2 мм окружающей ткани, что, в свою очередь, ограничивает воздействие радиотрейсера на близлежащие органы. Происходит нарушение биосинтеза гормонов ЩЖ, некроз фолликулов и кровеносных сосудов.

Эмпирический опыт, накопленный в результате применения РЙТ в разных возрастных группах в течение 80 лет, свидетельствует в пользу эффективности данного вида лечения, в том числе при лечении гипертиреоза. В большинстве случаев достичь стойкого гипотиреоза удается в течение 6-12 месяцев после лечения радиоактивным йодом [8-10]. Преимуществами РЙТ являются минимальные ограничения метода даже при наличии сопутствующих заболеваний и возможность неоднократного проведения. Также отдельно стоит отметить безопасность терапии радиоактивным йодом: согласно многочисленным данным литературы с описанием отдаленных последствий лечения, нет необходимости опасаться радиогенных эффектов РЙТ [11, 12].

Наследственные аномалии молекулы гемоглобина (обусловленные заменой одной или нескольких аминокислот в цепи глобина либо отсутствием участка цепи или ее удлинением) входят в число наиболее распространенных клинически значимых генетически обусловленных состояний [13]. При этом, по данным ВО3, примерно 7\% населения Земли являются носителями имеющих клиническую значимость аномальных вариантов гемоглобинов [14]. Всего известно около 1000 вариантов человеческого гемоглобина $[15,16]$, и ежегодно этот список только расширяется. В России в связи с миграцией населения также наблюдается тенденция к увеличению числа гемоглобинопатий, но в основном количественных (талассемии) и в меньшей степени качественных (аномальные гемоглобины), поскольку приезд групп населения из регионов, где распространена серповидноклеточная анемия, незначителен, а из стран бывшего СССР, эндемичных по талассемии, наоборот, значителен [17].

Существует несколько классификаций гемоглобинопатий. В Российской Федерации традиционно широко используется классификация, разработанная Л.И. Идельсоном. Согласно этой классификации, все гемоглобинопатии делятся:

1. на анемии, связанные с нарушением синтеза цепей глобина (в эту группу входят $\beta$-, $\beta \delta$-, a- и Lepore-талассемии);

2. анемии, связанные с нарушением структуры цепей глобина (в эту группу входят анемии, обусловленные носительством гемоглобинов):

а) изменяющих структуру в условиях гипоксии (S);

б) стабильных аномальных гемоглобинов (C, D, E и других);

в) нестабильных аномальных гемоглобинов (Köln, Savannah, Southhampton-Casper, Takoma, Belfast, Москва, Hasharon-Sinai-Sealy, Волга и других всего их известно более 50).

Большинство вариантов гемоглобинов никак себя не проявляют ни клинически, ни гематологически, в то время как некоторые из них, например Hb S, вызывают гемолитическую анемию или цианоз, как в случае c Hb Kansas. Другие являются нестабильными гемоглобинами, а наличие Нb М может приводить к метгемоглобинемии, и, наконец, присутствие Hb Ypsilanti, Hb Malmö благодаря повышенному сродству к кислороду способствует развитию полицитемии [18]. Таким образом, клинические проявления при гемоглобинопатиях, обусловленных нестабильными гемоглобинами, аминокислотные замещения в которых вызывают неустойчивость их молекулы к нагреванию, воздействию окислителей и других повреждающих факторов, варьируют в широких пределах, включая бессимптомное носительство. 
Что касается описанной в семьях евреев-ашкенази сначала в Израиле, а позже в США [19] гемоглобинопатии с наличием нестабильного гемоглобина Hasharon, где гистидин заменен на аспарагиновую кислоту в позиции a47 [20], то при этом относительно нетяжелом виде аномальности гемоглобина анемия может отсутствовать или иметь «мягкое» течение с так называемым оккультным и компенсированным гемолизом. В процентном отношении у гетерозигот Hb Hasharon составляет 16-19\%, что объясняется его небольшим синтезом, и в конечном итоге «защищает» гетерозиготных носителей данного аномального гемоглобина от клинически значимой гемолитической анемии [21]. Тем не менее при присоединении инфекции, повышении температуры тела, приеме оксидантов у аффектных лиц может возникнуть клинико-гематологическая картина гемолитической анемии.

О том, какими могут быть проявления при проведении РЙТ у носителей нестабильного Hb Hasharon, до сих пор нет ни одной публикации ни в России, ни за рубежом.

\section{КЛИНИЧЕСКИЙ СЛУЧАЙ}

Пациентка X., 53 года (1966 г.), поступила в отделение радионуклидной терапии ФГБУ «НМИЦ эндокринологии» 01.07.2019 с диагнозом « Диффузно-узловой токсический зоб (V - 8,6 мл). Тиреотоксикоз средней степени тяжести. Медикаментозный гипотиреоз» с целью проведения лечения радиоактивным йодом.

Жалобы при поступлении неспецифичны: общая слабость, быстрая утомляемость.

Из анамнеза известно, что тиреотоксикоз диагностирован в июле 2018 г., сразу инициирована медикаментозная терапия препаратом Тирозол в дозировке 20 мг. Со слов пациентки, на фоне однократной попытки отмены отмечался рецидив тиреотоксикоза. Пациенткой также предоставлена документация с результатами проведенных лабораторных и инструментальных исследований.

По данным сцинтиграфии щЖ с 99mТс-пертехнетатом от 06.03.2019 отмечается диффузно-неоднородное накопление РФП в правой доле, с зонами повышенного накопления РФП в нижней половине размерами до 7 мм в диаметре, повышение общего индекса захвата РФП (2,7\%).

По данным ультразвукового исследования ЩЖ

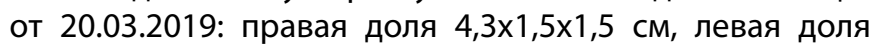
13,9x1,4x1,4 см, перешеек 0,3 см, общий объем - 8,6 мл. В средней трети правой доли определяется образование средней эхогенности, неоднородной структуры, с четкими контурами, размерами 0,8×0,6×0,6 см. При цветовом допплеровском картировании васкуляризация незначительно усилена. Региональные лимфатические узлы не изменены. Заключение: признаки правостороннего узлового зоба (TIRADS 2) на фоне аутоиммунного поражения ткани ЩЖ.

Результаты лабораторной диагностики от 03.06.2019 на фоне проводимой тиреостатической терапии: ТТГ 5,57 мМЕ/л (0,35-5,5), Т4Св - 11,42 пмоль/л (10,5-23,2), ТЗсв. - 3,32 пмоль/л (3,5-6,5), АТ к ТГ - 20,0 МЕ/мл, АТ к ТПО - 12,6 МЕ/мл, АТ к рТТГ - 1,41 МЕ/л (0-1,75). Тирозол был отменен 22.06.2019 в связи с предстоящим лечением радиоактивным йодом, повторные лабораторные исследования непосредственно перед РЙТ не проводились.

Перед принятием решения о возможности проведения терапии радиоактивным йодом пациентка была направлена на консультацию к гематологу ФГБУ «НМИЦ эндокринологии» Минздрава России для определения возможных противопоказаний к лечению в связи с наличием у нее в анамнезе наследственной формы гемоглобинопатии.

При осмотре гематологом 23.05.2019 жалоб активно не предъявляла. Со слов пациентки, около 20 лет назад у нее выявили наличие аномального гемоглобина $G$, но никакого дообследования с тех пора не проходила. Дочь и сын пациентки также ранее обследовались в связи с подозрением на наличие серповидноклеточной анемии, которая не подтвердилась. При уточнении семейного анамнеза было выяснено, что дедушка по отцу пациентки - еврей-ашкенази, в возрасте 4-5 лет тяжело переболел малярией.

В анамнезе у пациентки хронический холецистит, на момент осмотра вне обострения. Иногда отмечалось незначительное повышение уровня билирубина в сыворотке крови, при этом самые высокие значения отмечались в 2018 г., когда общий билирубин составлял 27,9 мкмоль/л, а прямой билирубин - 15,4 мкмоль/л. Анемии никогда не выявлялось. В представленной пациенткой гемограмме от 11 апреля 2019 г. эритроциты составляли 4,83×1012/л, гемоглобин - 141 г/л, средний объем эритроцитов - 84 фл. В биохимическом анализе сыворотки крови общий билирубин 25,8 мкмоль/л, свободный билирубин 22,4 мкмоль/л. Уровни креатинина, мочевины, ЛДГ, ЩФ, АЛТ, АСТ, амилазы, глюкозы, общего белка в пределах нормальных значений. В общем анализе мочи без признаков отклонений от нормы.

Было назначено дообследование: развернутый анализ крови, развернутый биохимический анализ крови, электрофорез гемоглобина, тест на распределение эритроцитов по плотности (РЭПП), УЗИ печени, желчного пузыря, селезенки.

По данным дообследования: в гемограмме эритроциты 4,33×1012/л, гемоглобин - 131 г/л, средний объем эритроцитов 85 фл, ретикулоциты 1,04\%, при просмотре мазков телец Гейнца (Heinz) в эритроцитах не выявлено; в биохимическом анализе сыворотки крови общий билирубин - 24,2 мкмоль/л, связанный билирубин 4,8 мкмоль/л, свободный билирубин - 19,4 мкмоль/л, железо - 10,2 мкмоль/л, АЛТ - 22 ед/л, АСТ - 27 ед/л, ГГТ - 18 ед/л, ЩФ - 195 ед/л, глюкоза - 5,0 ммоль/л, общий белок - 73 г/л, мочевина - 5,0 ммоль/л, креатинин 67 мкмоль/л, мочевая кислота - 237 мкмоль/л, холестерин - 6,01 ммоль/л; по данным УЗИ органов брюшной полости и почек - без признаков патологии за исключением УЗИ-признаков застоя желчи в желчном пузыре, размеры и эхоструктура селезенки и печени нормальные; по электрофорезу гемоглобина 27.05.2019 была выявлена патологическая фракция HbS, составляющая 17\% (рис. 1), а по данным исследования РЭПП было выявлено снижение средней плотности эритроцитов, при этом тяжелая и легкая фракции были в пределах нормы (рис. 2).

По результатам дообследования пациентка была направлена на дополнительную консультацию 


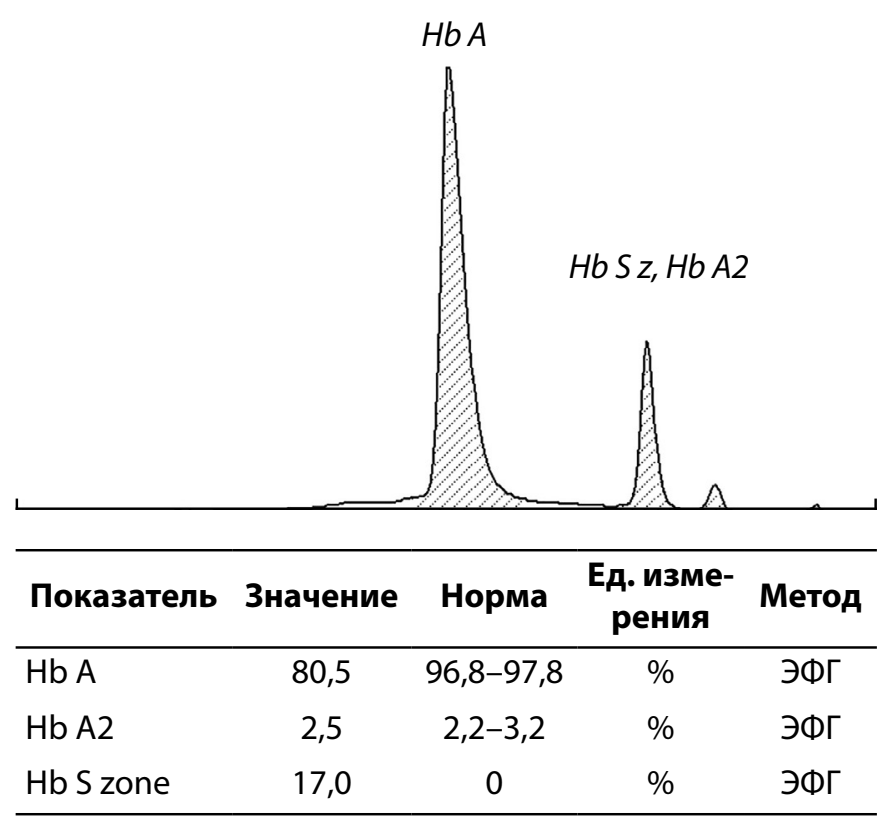

Рисунок 1. Результаты электрофореза гемоглобина. Выявлена фракция патологического гемоглобина S.

в ФГБУ «НМИЦ гематологии» Минздрава России, где было рекомендовано проведение анализа на наличие точечных мутаций сначала в генах $\alpha$ - и $\beta$-глобина. Во втором случае никаких мутаций выявлено не было, а в первом случае ДНК-диагностика, проведенная при помощи определения первичной структуры всех функционально важных фрагментов генов альфа-цепей глобина, показала наличие в гене HBA2 миссенс-мутации CD47 GAC>CAC (Asp47His) в гетерозиготном состоянии, приводящей к образованию аномального гемоглобина $\mathrm{Hb}$ Hasharon (другие названия - Sinai, Sealy, L-Ferrara).

Таким образом, был верифицирован диагноз наследственной гемоглобинопатии с наличием (17\%) нестабильного гемоглобина Hasharon-Sinai-Sealy $a_{2}^{47 а с п \rightarrow г и с ~} \beta_{2}$ в гетерозиготной форме. Признаков гемолитической анемии выявлено не было. Пациентке было рекомендовано полностью исключить возможность употребления сульфаниламидных препаратов, аспирина, седурала, фенацетина, нитрофурантоина, производных витамина К и, хотя это звучит казуистически, противомалярийных средств. Абсолютных противопоказаний к проведению РЙТ выявлено не было.

01.07.2019 проведено индивидуальное дозиметрическое планирование с расчетом оптимальной активно-

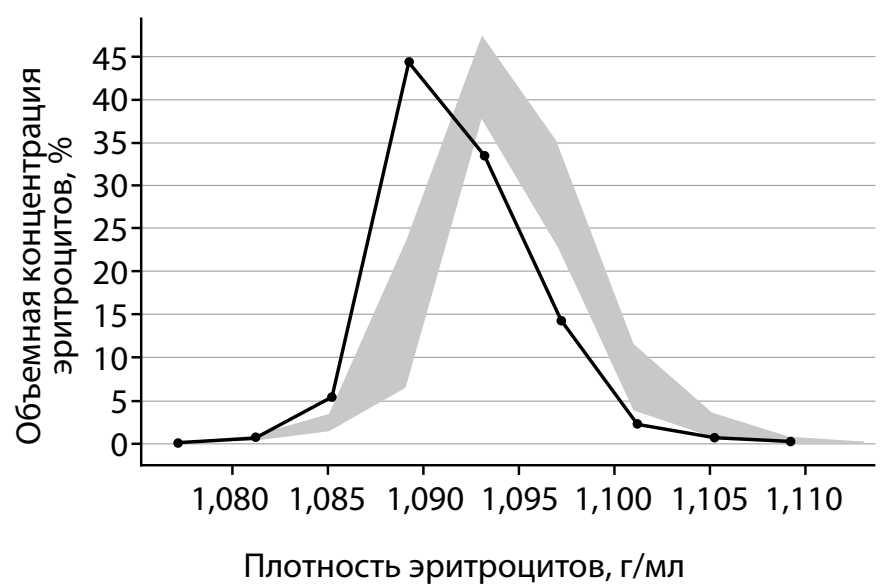

$\longrightarrow$ 28.05.2019 Норма 24,0-26,0

Рисунок 2. Исследование распределения эритроцитов по плотности.

Заключение: выявляется снижение средней плотности эритроцитов. Тяжелая и легкая фракции в пределах нормы.

сти, 02.07.2019 проведена радионуклидная терапия І-131 активностью 400 МБк. Осложнений и реакций на введение РФП зафиксировано не было.

За время нахождения пациентки в стационаре отмечалось удовлетворительное самочувствие, жалоб активно не предъявляла. 04.07.2019 пациентка была выписана из отделения радионуклидной терапии, показатели внешнего излучения при дозиметрии соответствовали нормам радиационной безопасности (НРБ) 99/2009, мощность дозы излучения от тела на расстоянии 1 м 19,9 мк3в/ч (остаточный фон ионизирующего излучения, допустимый НРБ).

В посттерапевтическом периоде проводился ежемесячный контроль уровня ТТГ, свТ3, свТ4 после РЙТ; уровня АТ-рТТГ и УЗИ щитовидной железы - через 3 и 6 месяцев, а также уровня общего и прямого билирубина.

Сначала контроль лабораторных параметров проводился 1 раз в неделю, а далее один раз в месяц. Можно констатировать, что через 6 месяцев после РЙТ никаких признаков гемолитической анемии у пациентки с гемоглобинопатией с гетерозиготным носительством аномального гемоглобина Hasharon не развилось. Ежемесячная динамика лабораторных показателей в посттерапевтическом периоде представлена в таблице 1.

Таблица 1. Динамика лабораторных показателей после радиойодтерапии

\begin{tabular}{|c|c|c|c|c|c|c|c|c|}
\hline $\begin{array}{c}\text { Время } \\
\text { после } \\
\text { РЙТ }\end{array}$ & $\begin{array}{c}\text { ТТГ, } \\
\text { мМЕ/л }\end{array}$ & $\begin{array}{c}\text { свТ3, } \\
\text { пмоль/л }\end{array}$ & $\begin{array}{c}\text { свТ4, } \\
\text { пмоль/л }\end{array}$ & $\begin{array}{c}\text { Ат-рТТГ, } \\
\text { Ме/л }\end{array}$ & $\begin{array}{c}\text { Объем } \\
\text { щитовидной } \\
\text { железы, см } \\
\text { (УЗИ) }\end{array}$ & $\begin{array}{c}\text { L-тироксин, } \\
\text { мкг }\end{array}$ & $\begin{array}{c}\text { Билирубин } \\
\text { общий, } \\
\text { мкмоль/л }\end{array}$ & $\begin{array}{c}\text { Билирубин } \\
\text { прямой, } \\
\text { мкмоль/л }\end{array}$ \\
\hline $1 \mathrm{мec}$ & 0,11 & 6,16 & 19,58 & - & - & - & 19,5 & 3,0 \\
\hline 2 мес & $8,36 / 66,08$ & $3,28 / 1,84$ & 5,9 & - & - & - & 21,5 & 2,9 \\
\hline $3 \mathrm{мес}$ & 43,49 & 1,82 & 7,4 & 8,19 & 4,4 & $50 / 100$ & 18,5 & 1,7 \\
\hline 4 мес & 1,54 & 4,49 & 22,14 & 1,54 & - & 100 & 25,3 & 8,3 \\
\hline $5 \mathrm{mec}$ & 0,38 & 4,55 & 20,25 & - & - & 100 & 16,7 & 3,1 \\
\hline $6 \mathrm{mec}$ & 0,9 & 3,3 & 14,23 & 32,9 & 3,5 & 100 & 20,8 & 5,9 \\
\hline
\end{tabular}




\section{ОБСУЖДЕНИЕ}

Исходя из проведенного нами анализа научной литературы, можно заявлять, что подобного клинического опыта в мире на данный момент не зарегистрировано, что объяснимо с учетом эксклюзивности подобной гемоглобинопатии, особенно в сочетании с тиреотоксикозом. Учитывая все вышесказанное, после верификации диагноза наследственной гемоглобинопатии с гетерозиготным носительством нестабильного гемоглобина Hasharon каких-либо признаков гемолитической анемии у пациентки не было выявлено. Следовательно, не было противопоказаний к РЙТ, после проведения которой пациентка наблюдалась гематологом: клинических признаков гемолиза эритроцитов сразу после процедуры введения радиоактивного йода зафиксировано не было. По результатам последующего лабораторного контроля (общий анализ крови, биохимический анализ крови, общий анализ мочи) признаков гемолитической анемии отмечено не было.

\section{ЗАКЛЮЧЕНИЕ}

В описанном нами клиническом случае терапия радиоактивным йодом тиреотоксикоза оказалась эффективной и не сопровождалась осложнениями и усугублением сопутствующей патологии. Любой клинический случай терапии радиоактивным йодом у пациентов с гемоглобинопатиями (или другой значимой патологией) должен рассматриваться индивидуально с учетом возможных рисков по результатам проведенного предварительно обследования.

\section{ДОПОЛНИТЕЛЬНАЯ ИНФОРМАЦИЯ}

Источник финансирования. Поисково-аналитическая работа и подготовка статьи проведены на личные средства авторского коллектива.

Согласие пациента. Персональные медицинские данные опубликованы с письменного согласия пациентов.

Конфликт интересов. Все авторы декларируют отсутствие явных и потенциальных конфликтов интересов, связанных с публикацией настоящей статьи.

Участие авторов. Все авторы принимали участие в наблюдении пациента, внесли значимый вклад в подготовку статьи, прочли и одобрили финальную версию перед публикацией.

\section{СПИСОК ЛИТЕРАТУРЫ | REFERENCES}

1. De Leo S, Lee SY, Braverman LE. Hyperthyroidism. Lancet. 2016;388(10047):906-918. doi: 10.1016/50140-6736(16)00278-6.

2. Трошина Е.А., Ванушко В.Э., Румянцев П.О, и др. Федеральные клинические рекомендации Российской ассоциации эндокринологов по диагностике и лечению токсического зоба // Клиническая и экспериментальная тиреоидология. 2014. -T.10. - №3. - C. 8-19. [Troshina EA, Sviridenko NYu, Vanushko VE, et al. Russian association of endocrinologists clinical practice guidelines for thyrotoxicosis diagnosis and treatment. Clinical and experimental thyroidology. 2014;10(3):8-19. (In Russ).] doi: $10.14341 /$ ket201438.19.

3. Vanderpump MP. The epidemiology of thyroid disease. Br Med Bull. 2011;99:39-51. doi: 10.1093/bmb/ldr030.

4. Аметов А.С., Кондратьева Л.В., Бугова Л.А. Болезнь Грейвса и функциональная автономия щитовидной железы в регионе с легким йодным дефицитом // Клиническая и экспериментальная тиреоидология. - 2011. - T.7. — №4. - C. 51-55. [Ametov AS, Kondrat'eva LV, Bugova LA. Graves" disease and thyroid functional autonomy in the region of mild iodine deficiency. Clinical and experimental thyroidology. 2011;7(4):51-55. (In Russ).]

5. Goichot B, Caron P, Landron F, et al. Clinical presentation of hyperthyroidism in a large representative sample of outpatients in France: Relationships with age, aetiology and hormonal parameters. Clin Endocrinol (Oxf). 2016;84(3):445-451. doi: $10.1111 /$ cen. 12816 .

6. Devereaux D, Tewelde SZ. Hyperthyroidism and thyrotoxicosis. Emerg Med Clin North Am. 2014;32(2):277-292. doi: 10.1016/j.emc.2013.12.001.

7. Ross DS, Burch HB, Cooper DS, et al. 2016 American Thyroid Association Guidelines for diagnosis and management of hyperthyroidism and other causes of thyrotoxicosis. Thyroid. 2016;26(10):1343-1421. doi: 10.1089/thy.2016.0229.

8. Sarkar SD. Thyroid gland. In: Elgazzar AH. The pathophysiologic basis of nuclear medicine. Springer Nature Switzerland AG; 2006. P. 209-221.

9. Van Mohamed WM, Sayuti SC, Draman N. Hypothyroidism and its associated factors after radioactive iodine therapy among patients with hyperthyroidism in the Northeast Coast State of Malaysia. J Taibah Univ Med Sci. 2018;13(5):432-437. doi: 10.1016/j.jtumed.2018.06.004.

10. Kaplan MM, Meier DA, Dworkin HJ. Treatment of hyperthyroidism with radioactive iodine. Endocrinol Metab Clin North Am. 1998;27(1):205-223. doi: 10.1016/50889-8529(05)70307-8.
11. Румянцев П.О., Кияев А.В. Шеремета М.С., и др. Радиойодтерапия тиреотоксикоза у детей и подростков. Показания, эффективность и безопасность. Обзор литературы // Эндокринная хирургия. 2016. - T.10. — №4. — C. 6-12. [Rumiantsev PO, Kiyaev AV, Sheremeta MS, et al. Radioiodine therapy of thyrotoxicosis in children and adolescents. Indications, efficacy and safety. Literature review. Endocrine surgery. 2016;10(4):6-12. (In Russ).] doi: 10.14341/serg201646-12

12. Fard-Esfahani P, Beiki D, Fallahi B, et al. Radioiodine therapy for hyperthyroidism. Iranian Journal of Nuclear Medicine. 2011;19(2):1-12. doi: 10.1056/nejmc1103014

13. Modell B, Darlison M. Global epidemiology of haemoglobin disorders and derived service indicators. Bull World Health Organ. 2008:86(6):480-487. doi: 10.2471/BLT.06.036673.

14. Kohne E. Hemoglobinopathies: clinical manifestations, diagnosis, and treatment. Dtsch Arzteb/ Int. 2011;108(31-32):532-540. doi: 10.3238/arztebl.2011.0532.

15. Hardison RC, Chui DH, Giardine B, et al. HbVar: A relation database of human hemoglobin variants and thalassemia mutations at the globin gene server. Hum Mutat. 2002;19(3):225-233. doi: 10.1002/humu.10044.

16. Patrinos GP, Wajcman H. Recording human globin gene variation. Hemoglobin. 2004;28(2):v-vii.

17. Лохматова М.Е., Сметанина Н.С., Финогенова Н.А. Эпидемиология гемоглобинопатий в Москве // Педиатрия. Журнал им. Г.Н. Сперанского. - 2009. - Т.87. - №4. - С. 46-49. [Lokhmatova ME, Smetanina NS, Finogenova NA. Epidemiologiya gemoglobinopatii v Moskve. Pediatriya. Zhurnal im. G.N. Speranskogo. 2009;87(4):46-50. (In Russ).]

18. Moradkhani K, Prehu C, Old J, et al. Mutations in the paralogous human alpha-globin genes yielding identical hemoglobin variants. Ann Hematol. 2009;88(6):535-543. doi: 10.1007/s00277-008-0624-3.

19. Halbrecht I, Isaacs WA, Lehmann $\mathrm{H}$, et al. Hemoglobin hasharon (alpha-47 aspartic acid-histidine). Isr J Med Sci. 1967;3(6):827-831 .

20. Schneider RG, Ueda S, Alperin JB, et al. Hemoglobin sealy (alpha 2-47His-beta 2): a new variant in a Jewish family. Amer J Hum Genet. 1968;20(2):151-156.

21. Carache S, Mondzac AM, Gessner U. Hemoglobin Hasharon (alpha-2-47 his(CD5)beta-2): a hemoglobin found in low concentration. J Clin Invest. 1969:48(5):834-847. doi 10.1172/JCl106041. 


\section{ИНФОРМАЦИЯ ОБ АВТОРАХ [AUTHORS INFO]}

*Дзейтова Дали Султановна [Dali S. Dzeytova, MD]; адрес: 117036 Москва, ул. Дм. Ульянова, д. 11, к. 2 [address: 11 bld 2, Dm. Ulyanova street, 117036 Moscow, Russia]; ORCID: https://orcid.org/0000-0003-3071-4314; eLibrary SPIN-код: 8761-7619; e-mail: dzdali@gmail.com

Шкляев Станислав Сергеевич [Stanislav S. Shklyaev, MD];

ORCID: https://orcid.org/0000-0002-9215-4984, eLibrary SPIN-код: 2975-4121; e-mail: staniss1@yahoo.com

Румянцев Павел Олегович, д.м.н. [Pavel O. Rumyantsev, MD, PhD]; ORCID: https://orcid.org/0000-0002-7721-634X;

eLibrary SPIN-код: 7085-7976; e-mail: pavelrum@gmail.com

Шеремета Марина Сергеевна, к.м.н. [Marina S. Sheremeta, MD, PhD]; ORCID: https://orcid.org/0000-0003-3785-0335;

eLibrary SPIN-код: 7845-2194; e-mail: marina888@yandex.ru

Трухин Алексей Андреевич [Alexey A. Trukhin]; ORCID: https://orcid.org/0000-0001-5592-4727;

eLibrary SPIN-код: 4398-9536; e-mail: alexey.trukhin12@gmail.com

Цветаева Нина Валентиновна [Nina V. Tsvetaeva], eLibrary SPIN-код 3420-6275; https://orcid.org/0000-0002-0977-215X; e-mail: tsvetaevan51@gmail.com

Кожедуб Евгений Евгеньевич [Evgenii E. Kozhedub]; ORCID: https://orcid.org/0000-0001-9422-1450;

eLibrary SPIN-код: 2169-8276; e-mail: kogedyb1@ya.ru

\section{ЦИТИРОВАТЬ:}

Дзейтова Д.С., Шкляев С.С., Румянцев П.О., Шеремета М.С., Трухин А.А., Цветаева Н.В., Кожедуб Е.Е. Терапия радиоактивным йодом тиреотоксикоза у пациентки с сопутствующей гемоглобинопатией с носительством нестабильного гемоглобина HASHARON // Проблемы эндокринологии. — 2020. — Т. 66. — №3. - С. $27-32$. doi: https://doi.org/10.14341/probl12459

\section{TO CITE THIS ARTICLE:}

Dzeytova DS, Shklyaev SS, Rumyantsev PO, Sheremeta MS, Trukhin AA, Tsvetaeva NV, Kozhedub EE. Radioiodine therapy outcome in toxic multinodular goiter patient with concomitant hereditary Hasharon hemoglobinopathy. Problems of Endocrinology. 2020; 66(3):27-32. doi: https://doi.org/10.14341/probl12459 